\title{
Visual Analytics to Optimize Patient-Population Evidence Delivery for Personalized Care
}

\author{
Ketan K. Mane, Phillips Owen, \\ Charles Schmitt, Kirk Wilhelmsen \\ RENCl, UNC-Chapel Hill \\ 100 Europa Dr, Suite 540, Chapel Hill, NC \\ +1-919-445-9703 \\ \{kmane, powen, cschmitt, kirk\}@renci.org
}

\author{
Kenneth Gersing, Ricardo Pietrobon, \\ Igor Akushevich \\ Duke University \\ Duke University Medical Center, Durham, NC \\ +1-919-684-0103 \\ \{kenneth.gersing, rpietro, ia6\} @duke.edu
}

\begin{abstract}
Electronic medical records (EMR) can be used to identify cohorts of patients who are clinically comparable to an individual patient. In this paper, we describe an approach that applies visual analytics to EMR data to describe the clinical course for an individual patient, display outcomes for a comparable cohort stratified by treatment, and generate predictions regarding a patient's clinical course based on treatment options. The visual display of information is designed to help clinicians choose among alternative therapies based on the EMR-derived outcomes of the cohort.
\end{abstract}

\section{Categories and Subject Descriptors \\ [Programming Languages]: Java}

\section{General Terms}

Design, Human Factors.

\section{Keywords}

Visual analytics, Decision Support, Comparative Evidence, Personalized care, Electronic Medical Records

\section{INTRODUCTION}

In clinical practice, physicians typically rely on personal experience, and guidelines and recommendations developed from the results of clinical trials. In complex cases, the experience or expertise of a physician may not be sufficient to identify the most appropriate choice from among many treatment options $[4,10]$. In addition, relevant clinical trials are often not available for complex or rare conditions, and the real-world relevance of clinical trial results has been questioned [10].

The widespread adoption of electronic medical records (EMR) offers an opportunity to leverage information derived from aggregated, real-world patient data to bridge the evidence gap and

Permission to make digital or hard copies of all or part of this work for personal or classroom use is granted without fee provided that copies are not made or distributed for profit or commercial advantage and that copies bear this notice and the full citation on the first page. To copy otherwise, or republish, to post on servers or to redistribute to lists, requires prior specific permission and/or a fee.

$B C B^{\prime} 13$, September 22-25, 2013, Washington, DC, USA.

Copyright 2013 ACM 978-1-4503-2434-2/13/09 ...\$15.00. better guide clinical decisions regarding patient care [7, 17].

Digital support is required to use EMR data effectively because clinical decisions are made in a setting where it is impractical to rapidly draw conclusions from large volumes of EMR data. Prior efforts to use EMR data for clinical decision support have been limited and typically have not incorporated a comparative effectiveness approach to draw treatment insights [9, 11, 14-16]. To be useful in the clinical setting, physicians need a user interface (UI) that has views that complement their typical workflow tasks. A primary requirement for UI design in an approach that incorporates comparative effectiveness is that it displays patient-specific information that describes: demographic and diagnostic features; clinical course with past and current therapeutic interventions; response patterns in the clinical course of a comparative cohort; and predicted outcomes with current and alternative treatments.

In this paper, we report the application of visual analytics in a data-driven approach that leverages EMR data from a cohort of patients with major depressive disorder (MDD) to evaluate treatment options for an individual patient and thereby personalize care. Visual analytics aims to use analytical reasoning to facilitate the development and use of interactive visual displays or interfaces [5, 12]. We believe that visual analytics is critical to transform data-to-actionable information and display the evidence in a form that readily bridges the evidence gap in patient care. We applied a visual analytics approach to build a single UI dashboard that can be optimized to deliver patient, as well as population level, evidence. The dashboard offers flexibility in that viable treatment options can be quickly narrowed down to just a few choices from many possibilities. As an extension of our previous model [8], the updated UI described herein was designed to incorporate the clinician's workflow, as well as provide evidence from a comparative cohort, to better personalize treatment decisions regarding an individual patient. Our redesigned UI provides treatment-related evidence on three levels: a) an at-aglance overview of a patient's treatment-response profile; b) aggregated evidence from a comparable population on treatmentrelated outcomes to provide predictive insight into potential treatment outcomes for an individual patient; and c) contextualized treatment guidelines to assist the clinician at the point of care.

This manuscript is organized as follows: Section 2 provides a brief overview of the psychiatric dataset used in this report; Section 3 highlights the individual building blocks we used to process and transform the data and thereby provide data views for 
decision support; Section 4 provides details about the UI data views, including interactivity and predictive insight; and Section 5 concludes with a discussion of the current results and future directions.

\section{DATASET OVERVIEW}

We used anonymized patient data from the MindLinc EMR system, which is the largest de-identified, psychiatry-focused, outcome-based, data warehouse in the United States. It is a clinically representative sample of data collected during customary psychiatric practice [2]. To test the feasibility of our data-driven approach, we limited our focus to psychiatric patients with Major Depressive Disorder (MDD) as their primary diagnosis. Available data were curated to remove data inconsistencies (typos, missing data) and normalized. The resulting MDD dataset contained data on 33,536 patient encounters from 3,016 unique patients. Curated data attributes included: prescribed medications; comorbid conditions; demographics (race, gender, age); visit type (inpatient, outpatient, emergency), and treatment outcomes. Within MindLinc, the treatment outcome is recorded using a Clinical Global Impression (CGI) score, which was developed and validated for use by the National Institute of Mental Health and has been applied in all FDA-regulated and most clinical trials $[1,3,6]$. The CGI score ranges from 1 through 7 , with 1 indicating 'no illness/maximum improvement' and 7 indicating 'severe illness/maximum worsening'.

\section{BUILDING BLOCKS FOR VISUAL ANALYTICS DECISION SUPPORT}

Figure 1 provides a schematic overview of the different building blocks underlying our UI, with computational approaches to process, summarize, and present the data. We categorized each component into 4 different layers, the details of which are described below.

\subsection{Data Layer}

This layer represents the raw de-identified EMR data from the MindLinc database. The data are loaded into a Microsoft SQL Server, with preservation of the MindLinc database table relationships.

\subsection{In-database Processing Layer}

In this layer, data cleaning techniques are applied to the raw data to check for inconsistencies (typos, missing data) and to correct them with successive and recursive quality checks. All data that meet data quality standards are stored in a Processed Data Table and made available for subsequent data mining and eventual visual display.

\subsection{Data-To-Discovery Layer}

Data mining algorithms and predictive modeling techniques are applied to further process the data before the information is displayed on the UI. Data Analytics and Integration are used to process the curated data, summarize and aggregate the data on both patient and population levels, and perform the predictive modeling analytics. The Data Linking and Visualization component is responsible for collecting data for different visual data views and linking them together for performance enhancements at the UI level.

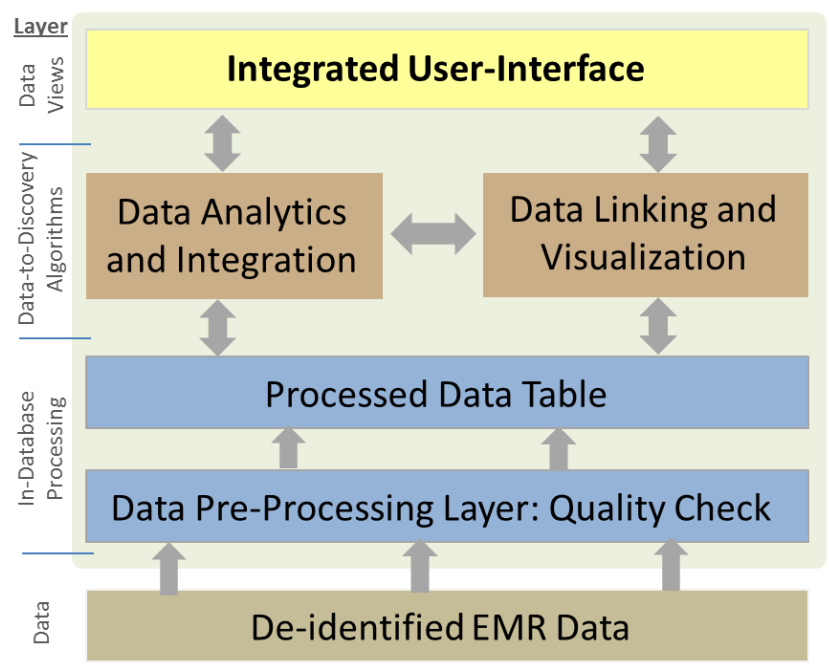

Figure 1. Schematic overview of the UI building blocks.

\subsection{Data Views Layer}

This layer includes various data views that are integrated into a single dashboard UI (figure 2). The Data Views layer supports integrated ad hoc data filtering techniques designed to specifically update and display multiple data views and the results of predictive modeling. The UI is optimized such that data filtering or changes made in one view are instantly updated in the other views.

\section{VISUAL ANALYTICS - UI APPLICATION}

Visual analytics were used to generate visual summaries from the patient and aggregated cohort EMR data. Figure 2 shows our dashboard UI, which is optimized to present different clinical characteristics for an individualized patient and evidence from a comparative cohort; this information is then contextualized for the target patient, as described below.

\subsection{Patient: Demographics and Diagnosis}

This data view (label 1) is configured to gain quick insight into the clinical status of an individual patient. The user enters the patient medical record number $(\mathrm{MRN}$, shown pseudo $\mathrm{MRN}=$ 175). The MRN is then used to identify a given patient from the Processed Data Table (section 3.2) and display that patient's demographic information (age, gender, race), along with the primary and secondary diagnoses.

\subsection{Patient: Longitudinal Profile of Treatment Outcomes with Different Medications}

This data view (label 2) is configured to provide insight into an individual patient's clinical course after different therapeutic interventions. Here, the $x$-axis shows the years of treatment, while the $y$-axis shows the CGI range. Patient outcome is plotted over time. Horizontal bars directly below the temporal treatment outcome view show the treatment time-spans for therapy and prescribed medications. Medication names are provided at a medication-class level due to the large number of available 


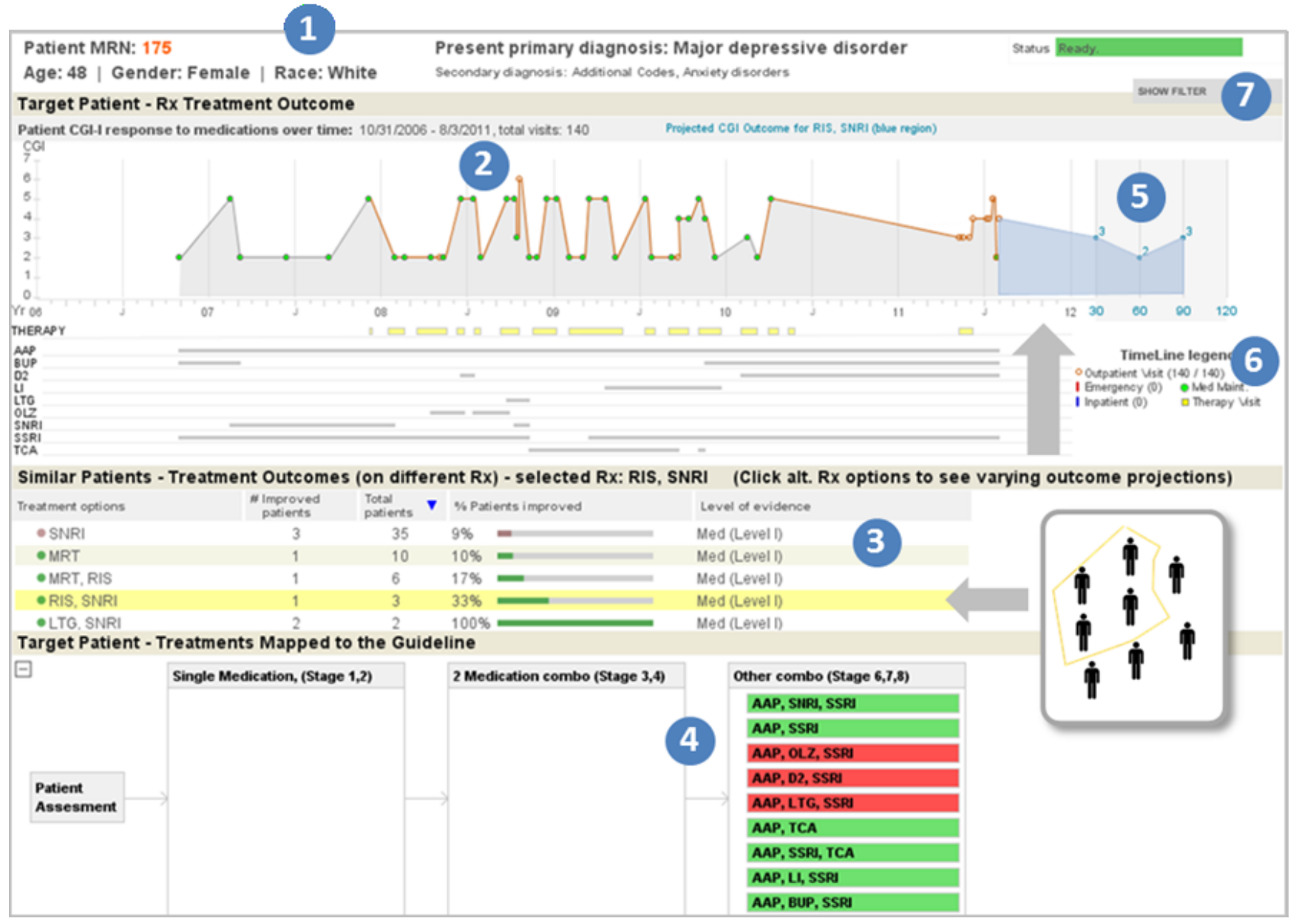

Figure 2. Dashboard-style UI with integrated data views. Label 1: Demographics and diagnosis; label 2: Longitudinal profile of treatment outcomes with different medications; label 3: Population-level evidence on treatments and outcomes; label 4: Treatments mapped to treatment guidelines; label 5: Predictive outcome trendline with selected treatment; label 6: Data filters to personalize evidence; and label 7: Visit type information.

medications; however, medication combinations are preserved and provided at a medication level.

\subsection{Comparative Cohort: Population-level Evidence on Treatments and Outcomes}

This data view (label 3 ) is configured to provide insight into the outcomes with alternative treatments, as determined from the aggregated data on the comparative cohort. Our approach depends on matching the target patient attributes to a comparable population. At run time, an individual patient's current treatment is used to establish the initial set of patients for the comparative cohort. Additional filtering is available to prune the cohort at different levels: demographics; medication class; and comorbid condition (figure 3). Aggregated data are summarized in multiple columns to provide a quick overview on: number of improved patients; number of total patients; and \% of patients showing improvement. A visual color cue is added to help distinguish between treatments with a greater success rate than others. Treatments associated with $\geq 10 \%$ patient improvement are highlighted in green, while other treatments are highlighted in red. User-selected treatment choice is highlighted in yellow, and predictive results with the selected treatment are shaded in blue in the patient course view (section 4.5).

\subsection{Patient: Treatments Mapped to Treatment Guidelines}

This data view (label 4) is designed to provide insight into how an individual patient's treatment history aligns with the treatment guideline evidence. To accomplish this feature, we built a decision engine based on logic within the MDD treatment guidelines [13]. The patient's medication and outcome data are used by the decision engine to map these data to a compressed representation in medication bins. Rectangular stacks in the bins show the medications taken, and the visual color cue provides information on treatment outcome with that medication, as suggested by the treatment guidelines. Green indicates a positive outcome, while red indicates a negative outcome. 


\subsection{Patient: Predictive Outcome Trendline with Selected Treatment}

This data view (label 5) is designed to provide insight into how likely an individual patient is to respond to a selected alternative treatment choice (section 4.3). Here, the predicted outcome is computed using data derived from the comparative population on outcomes with the selected alternative medication. Historic outcome data for each patient in the comparative population are aggregated, and a median CGI score is computed for each of the time points $(30,60,90,120$ days). Of note, this data view is dynamic; a change in outcome for the comparative population with the selected medication triggers automatic updates in the outcome trendline for an individual patient.

\subsection{Patient: Data Filters to Personalize Evidence}

A click on the filtering button (label 7) will bring up a filtering panel (figure 3), which contains a list of options to stratify the aggregated treatment evidence derived from the comparative population. Data filtering can be performed using several data attributes: demographics (gender, race, age); medication categories; and comorbid conditions. A "Y/N" (Yes/No) toggle allows the user to include or exclude these attributes.

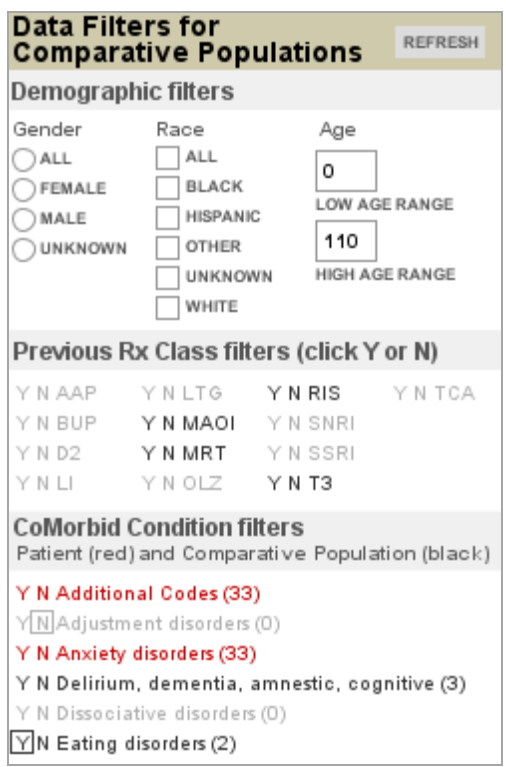

Figure 3. Default filter panel with data-attribute-level filters and "Y/N" (Yes/No) toggles for variable selection.

\subsection{Patient: Visit Type Information}

This view shows counts of the types of patient visits (outpatient, inpatient, emergency) for an individual patient (label 7), which are highlighted in brackets next to the name of the visit type. Therapy visits are highlighted along with patient's medications profile.

\subsection{Overall UI View}

At the overall UI view, all of the different data views are coupled together to function as an integrated whole. Of importance, any change in the data used to generate a single view automatically triggers a simultaneous and coordinated update to all of the other linked data views (section 3.4).

\section{CONCLUSION}

In conclusion, the visual analytics approach described herein offers the potential to bridge the skill set of the physician with the power of computer algorithms to rapidly derive useful clinical information from large clinical datasets. EMR data offer a wonderful opportunity to use real-world patient data to mitigate the limitations of controlled clinical trials and individual physician experience/expertise and harness the collective evidence required to generate insight on treatment options and outcomes. Our approach involves the application of visual analytics to EMR data to design interactive displays to augment a clinician's information-processing abilities while simultaneously reducing information overload and facilitating decision making. Our approach demonstrates the flexibility that visual analytics provides in the aggregation, visualization, and distillation of large volumes of EMR data at the UI level. We aim to further develop, evaluate, and validate the effectiveness of our visual analytics approach as a clinical decision-making tool for widespread adoption by physicians in various specialties and health care settings.

\section{ACKNOWLEDGMENTS}

This work was supported by funds from the North Carolina Translational and Clinical Sciences Institute, RENCI, the Agency for Healthcare Research and Quality (\# R21HS019023), and the National Science Foundation (\# 1117286). We acknowledge Chris Bizon from RENCI and Javed Mostafa from UNC-Chapel Hill for their effort and input during the initial development phases of this project. Technical editorial support was provided by Karamarie Fecho.

\section{REFERENCES}

[1] Busner, J. and Targum, S.D. 2007. The Clinical Global Impressions Scale. Psychiatry (Edgmont). 4, 7 (2007), 2837.

[2] Gersing, K. and Krishnan, R. 2003. Clinical Computing: Clinical Management Research Information System (CRIS). Psychiatric Services (Washington, D.C.). 54, 9 (Sep. 2003), 1199-1200.

[3] Guy, W. 1976. Clinical Global Impression: ECDEU assessment manual for psychopharmacology. U.S. Dept. of Health, Education, and Welfare, Public Health Service, Alcohol, Drug Abuse, and Mental Health Administration, National Institute of Mental Health, Psychopharmacology Research Branch, Division of Extramural Research Programs.

[4] Horn, S.D. and Gassaway, J. 2010. Practice based evidence: incorporating clinical heterogeneity and patientreported outcomes for comparative effectiveness research. Medical care. 48, 6 Suppl (Jun. 2010), S17-22.

[5] Keim, D.A. 2001. Visual exploration of large data sets. Commun. ACM. 44, 8 (Aug. 2001), 38-44.

[6] Lam, R.W. et al. 2006. Assessment Scales in Depression, Mania and Anxiety. Taylor \& Francis.

[7] Liu, J. et al. 2009. Toward a Fully De-identified Biomedical Information Warehouse. AMIA Annual Symposium Proceedings. 2009, (2009), 370-374.

[8] Mane, K.K. et al. 2012. VisualDecisionLinc: A visual analytics approach for comparative effectiveness-based clinical decision support in psychiatry. J. of Biomedical Informatics. 45, 1 (Feb. 2012), 101-106. 
[9] Plaisant, C. et al. 1998. LifeLines: using visualization to enhance navigation and analysis of patient records. Proceedings of the AMIA Symposium. (1998), 76-80.

[10] Rothwell, P.M. 2005. External validity of randomised controlled trials: "to whom do the results of this trial apply?" Lancet. 365, 9453 (Jan. 2005), 82-93.

[11] Shabtai, A. et al. 2006. An intelligent, interactive tool for exploration and visualization of time-oriented security data. Proceedings of the 3rd international workshop on Visualization for computer security (New York, NY, USA, 2006), 15-22.

[12] Thomas, J.J. and Cook, K.A. eds. 2005. Illuminating the Path: The Research and Development Agenda for Visual Analytics. National Visualization and Analytics Ctr.

[13] Trivedi MH, R.A. 2004. Clinical results for patients with major depressive disorder in the Texas Medication Algorithm Project. Archives of General Psychiatry. 61, 7 (Jul. 2004), 669-680.
[14] Wang, T.D. et al. 2008. Aligning temporal data by sentinel events: discovering patterns in electronic health records. Proceedings of the SIGCHI Conference on Human Factors in Computing Systems (New York, NY, USA, 2008), 457466.

[15] Wongsuphasawat, K. et al. LifeFlow: Visualizing an Overview of Event Sequences.

[16] Wongsuphasawat, K. and Gotz, D. 2012. Exploring Flow, Factors, and Outcomes of Temporal Event Sequences with the Outflow Visualization. IEEE Transactions on Visualization and Computer Graphics. 18, 12 (2012), 2659-2668.

[17] Yang, J.J. et al. 2008. SYRIAC: The systematic review information automated collection system a data warehouse for facilitating automated biomedical text classification. AMIA Symposium. (2008), 825-829. 